\title{
COMPACT AND WEAKLY COMPACT COMPOSITION OPERATORS ON BMOA
}

\author{
JUSSI LAITILA, PEKKA J. NIEMINEN, EERO SAKSMAN, AND HANS-OLAV TYLLI
}

\begin{abstract}
Any analytic map $\varphi$ of the unit disc $\mathbb{D}$ into itself induces a composition operator $C_{\varphi}$ on $B M O A$, mapping $f \mapsto f \circ \varphi$, where $B M O A$ is the Banach space of analytic functions $f: \mathbb{D} \rightarrow \mathbb{C}$ whose boundary values have bounded mean oscillation on the unit circle. We show that $C_{\varphi}$ is weakly compact on $B M O A$ precisely when it is compact on $B M O A$, thus solving a question initially posed by Tjani and by Bourdon, Cima and Matheson in the special case of VMOA. As a crucial step of our argument we simplify the compactness criterion due to Smith for $C_{\varphi}$ on $B M O A$ and show that his condition on the Nevanlinna counting function alone characterizes compactness. Additional equivalent compactness criteria are established. Furthermore, we prove the unexpected result that compactness of $C_{\varphi}$ on $V M O A$ implies compactness even from the Bloch space into $V M O A$.
\end{abstract}

\section{InTRODUCTION}

Let $\mathbb{D}$ be the open unit disc of the complex plane $\mathbb{C}$. The space $B M O A$ consists of the analytic functions $f: \mathbb{D} \rightarrow \mathbb{C}$ whose boundary values have bounded mean oscillation on the unit circle $\mathbb{T}$. Equivalently, $f$ belongs to $B M O A$ if and only if the seminorm

$$
|f|_{*}=\sup _{a \in \mathbb{D}}\left\|f \circ \sigma_{a}-f(a)\right\|_{H^{2}}
$$

is finite, where $\|\cdot\|_{H^{2}}$ is the standard norm of the Hardy space $H^{2}$ and $\sigma_{a}(z)=(a-$ $z) /(1-\bar{a} z)$ is the automorphism of $\mathbb{D}$ that exchanges the points 0 and $a$. Then $B M O A$ becomes a Banach space under the norm $\|f\|_{*}=|f(0)|+|f|_{*}$. Furthermore, VMOA is the closed subspace of $B M O A$ consisting of those functions $f$ whose boundary values have vanishing mean oscillation, or equivalently, which satisfy

$$
\lim _{|a| \rightarrow 1}\left\|f \circ \sigma_{a}-f(a)\right\|_{H^{2}}=0 .
$$

We refer e.g. to [G] and $[\mathrm{Z}]$ for more information on the spaces $B M O A$ and $V M O A$.

If $\varphi: \mathbb{D} \rightarrow \mathbb{D}$ is an analytic map, then the composition operator $C_{\varphi}$ induced by $\varphi$ is the linear map defined by $C_{\varphi} f=f \circ \varphi$ for all analytic functions $f: \mathbb{D} \rightarrow \mathbb{C}$. It is well known that $C_{\varphi}$ is always bounded from $B M O A$ into itself and that $C_{\varphi}$ preserves $V M O A$ if and only if $\varphi \in V M O A$; see e.g. [St], $\mathrm{AFP}$ and $[\mathrm{BCM}$. Composition operators have been intensively studied on various spaces of analytic functions, and we refer to $[\mathrm{CMc}$ or $[\mathrm{Sh}$ for more about the classical background.

Date: 20 January 2010.

2000 Mathematics Subject Classification. Primary 47B33; Secondary 30D50, 46E15, $47 \mathrm{~B} 07$.

The first author was supported by the Academy of Finland, project 118422. The second and third authors were supported by the Finnish CoE in Analysis and Dynamics Research, and by the Academy of Finland, projects $118422 \& 126420$ and $113826 \& 118765$, respectively. 
Recall that a linear operator is compact if it takes bounded sets into sets having a compact closure. The compactness of a composition operator $C_{\varphi}$ acting on $B M O A$ (or on its subspace $V M O A$ ) has been investigated by several authors and various kinds of characterizations are known; see e.g. [T], [BCM], [Sm], [MT], [WX], [L2], [W], [L3] and [WZZ]. In particular, Smith $\left[\mathrm{Sm}\right.$ proved that $C_{\varphi}$ is compact on BMOA if and only if $\varphi$ satisfies the following pair of conditions:

$$
\lim _{|\varphi(a)| \rightarrow 1} \sup _{0<|w|<1}|w|^{2} N\left(\sigma_{\varphi(a)} \circ \varphi \circ \sigma_{a}, w\right)=0,
$$

and for all $0<R<1$,

$$
\lim _{t \rightarrow 1} \sup _{\{a:|\varphi(a)| \leq R\}}\left|\left\{\zeta \in \mathbb{T}:\left|\left(\varphi \circ \sigma_{a}\right)(\zeta)\right|>t\right\}\right|=0 .
$$

Above $N(\psi, \cdot)$ denotes the Nevanlinna counting function of an analytic self-map $\psi$ of the disc, $\varphi(\zeta)$ is the radial limit of $\varphi$ for a.e. $\zeta$ on the unit circle $\mathbb{T}$, and $|E|$ stands for the normalized Lebesgue measure of sets $E \subset \mathbb{T}$. Recently the first author [L3] showed that (S1) is equivalent to the condition

$$
\lim _{|\varphi(a)| \rightarrow 1}\left\|\sigma_{\varphi(a)} \circ \varphi \circ \sigma_{a}\right\|_{H^{2}}=0,
$$

which is technically more convenient for our later purposes.

A well-known open problem concerning composition operators is that of characterizing the weak compactness of $C_{\varphi}$ on the non-reflexive spaces BMOA and VMOA. Recall that an operator is weakly compact provided it takes bounded sets into sets whose closure is compact in the weak topology of the space. For $C_{\varphi}$ acting on $V M O A$ this problem was explicitly posed in [T] and [BCM], and for the BMOA case it was stated in [L1, L2]. Partial results for VMOA were obtained in [MT] and [CM]. For instance, if $\varphi \in V M O A$ and $\varphi(\mathbb{D})$ is contained in a polygon inscribed in $\overline{\mathbb{D}}$ [MT, Cor. 5.4], or if $\varphi$ is univalent [CM, p. 940], then compactness and weak compactness are equivalent for $C_{\varphi}$ on $V M O A$. It is natural to conjecture that the same equivalence should persist for arbitrary symbols $\varphi$ even on $B M O A$, especially because a similar phenomenon is known to occur for composition operators on many other classical non-reflexive spaces, such as $H^{1}\left[\mathrm{~S}\right.$, $H^{\infty}$ (see e.g. [AGL]) and Bloch spaces [MM, LST].

In the present paper we provide a solution to the above problem. Our main result reads as follows:

Theorem 1. Let $\varphi: \mathbb{D} \rightarrow \mathbb{D}$ be an analytic map. Then the following conditions are equivalent:

(i) $C_{\varphi}: B M O A \rightarrow B M O A$ is compact.

(ii) $C_{\varphi}: B M O A \rightarrow B M O A$ is weakly compact.

(iii) $\varphi$ satisfies condition (S1).

(iv) $\varphi$ satisfies condition (L).

A key ingredient of our argument is the surprising result that condition (L) (and consequently also (S1) actually implies (S2). This result is proved in Section 2 Thus our work substantially clarifies and simplifies the existing compactness criteria for composition operators on BMOA. The proof of Theorem 1 is then completed by verifying that (iii) implies (iv). This step is carried out in Section 3 , where the 
argument is based on an idea of Lerbov [Le] on how to construct explicit isomorphic copies of the sequence space $c_{0}$ inside $V M O A$.

As a by-product the results of Section 2 answer a recent question of Wulan, Zheng and Zhu [WZZ]. Namely, it follows that the condition $\lim _{|a| \rightarrow 1}\left|\sigma_{a} \circ \varphi\right|_{*}=0$ is sufficient for the compactness of $C_{\varphi}$ on $B M O A$. The necessity was earlier observed by Wulan [W].

In Section 4 we further reformulate $(\mathbb{L})$ as a pseudo-hyperbolic mean oscillation condition for the boundary values of the symbol as follows:

$$
\frac{1}{|I|^{2}} \int_{I} \int_{I} \rho(\varphi(\zeta), \varphi(\xi))^{2}|d \zeta||d \xi| \rightarrow 0 \quad \text { as } \quad\left|\frac{1}{|I|} \int_{I} \varphi(\zeta)\right| d \zeta|| \rightarrow 1
$$

Here $\rho$ denotes the pseudo-hyperbolic metric, $I \subset \mathbb{T}$ is a boundary arc and the integration is with respect to the normalized Lebesgue measure on $\mathbb{T}$.

Section 5 collects together some related results in the $V M O A$ setting. We observe that the analogue of Theorem 1 holds on VMOA (that is, for symbols $\varphi \in V M O A$ ), where $(\mathrm{L})$ can be replaced by $\lim _{|a| \rightarrow 1}\left\|\sigma_{\varphi(a)} \circ \varphi \circ \sigma_{a}\right\|_{H^{2}}=0$. Moreover, we prove that one may substitute the genuine hyperbolic metric for the pseudo-hyperbolic metric in the $V M O A$ version of condition (A). As an unexpected consequence, $C_{\varphi}$ is compact on VMOA if and only if it is compact from the Bloch space to VMOA.

\section{Compactness Characterization}

In this section we prove that condition $(\mathrm{L})$ alone is enough to characterize the compactness of $C_{\varphi}$ on the space $B M O A$. It is known that $(\mathrm{L})$ is equivalent to Smith's first condition (S1); see [L3, Remark 3.3]. Thus, in view of Smith's compactness criterion consisting of the pair (S1) and (S2), our work reduces to showing that (S2) is actually implied by (피 $)$, or by $(\underline{\mathrm{L}})$ :

Theorem 2. Condition $(\underline{\mathrm{L}}$ implies $(\underline{\mathrm{S} 2})$ for any analytic map $\varphi: \mathbb{D} \rightarrow \mathbb{D}$. Hence $C_{\varphi}: B M O A \rightarrow B M O A$ is compact if and only if $(\mathbb{L})$ holds.

We mostly work with $(\mathbb{L})$ because it is technically very convenient for our arguments and also allows for quite appealing reformulations in terms of the boundary values of $\varphi$. In particular, by expressing the $H^{2}$ norm as an $L^{2}$ norm on $\mathbb{T}$ and performing a change of variable using the automorphism $\sigma_{a}$, we get

$$
\begin{aligned}
\left\|\sigma_{\varphi(a)} \circ \varphi \circ \sigma_{a}\right\|_{H^{2}}^{2} & =\int_{\mathbb{T}} \rho\left(\varphi\left(\sigma_{a}(\zeta)\right), \varphi(a)\right)^{2}|d \zeta| \\
& =\int_{\mathbb{T}} \rho(\varphi(\zeta), \varphi(a))^{2} P_{a}(\zeta)|d \zeta|,
\end{aligned}
$$

where $P_{a}(\zeta)=\left(1-|a|^{2}\right) /|\zeta-a|^{2}$ is the Poisson kernel for $a \in \mathbb{D}$ and $\rho(z, w)=$ $|z-w| /|1-\bar{w} z|$ denotes the pseudo-hyperbolic distance in $\overline{\mathbb{D}}$ (observe that $\rho$ extends to the boundary $\mathbb{T}$ in a natural way if we agree that $\rho(z, z)=0$ for $z \in \mathbb{T})$. Thus (L) can be seen as a kind of vanishing mean oscillation condition with respect to the pseudo-hyperbolic metric. We will elaborate on this point further in Section 4.

It is useful to observe that if $\varphi$ satisfies condition (L), or equivalently (S1), then one has $|\varphi|<1$ a.e. on $\mathbb{T}$. This can be checked by a straightforward density point argument. 
The proof of Theorem 2 depends on the following lemma, which exhibits a uniform density estimate for Lebesgue measurable sets on $\mathbb{T}$. Since we have been unable to find a reference for this kind of result, we include a proof. Here and elsewhere in the text we use the following notation for closed arcs of $\mathbb{T}$ : when $r e^{i \theta} \in \mathbb{D}$ with $0 \leq r<1$, set

$$
I\left(r e^{i \theta}\right)=\left\{e^{i t}:|t-\theta| \leq \pi(1-r)\right\} .
$$

Thus $I\left(r e^{i \theta}\right)$ denotes the arc of $\mathbb{T}$ whose midpoint is $e^{i \theta}$ and (normalized) length $\left|I\left(r e^{i \theta}\right)\right|=1-r$.

Lemma 3. Suppose that $E \subset \mathbb{T}$ is a measurable set with $|E|>0$. Then there is a measurable set $E^{\prime} \subset E$ such that $\left|E^{\prime}\right|>0$ and

$$
\frac{|I(r \zeta) \cap E|}{|I(r \zeta)|} \geq \frac{1}{8}|E|
$$

for every $0 \leq r<1$ and $\zeta \in E^{\prime}$.

Proof. We say that an arc $I(a) \subset \mathbb{T}$ is dyadic if $a=\left(1-2^{-n}\right) \exp \left(2 \pi i k / 2^{n}\right)$ for some $n \geq 0$ and $0 \leq k \leq 2^{n}-1$. Note that any pair of dyadic arcs either are nested or have disjoint interiors.

Put $\lambda=1-\frac{1}{2}|E| \in\left[\frac{1}{2}, 1\right)$, and let $C$ be the set of all points $\zeta \in \mathbb{T}$ for which there exists a dyadic arc $I$ containing $\zeta$ and satisfying $\left|I \cap E^{c}\right|>\lambda|I|$, where $E^{c}=\mathbb{T} \backslash E$. Since for each $\zeta \in C$ there is a maximal one (in terms of inclusion) among such dyadic arcs, we have $C=\bigcup_{j} I_{j}$, where the $I_{j}$ 's are dyadic arcs with disjoint interiors satisfying $\left|I_{j} \cap E^{c}\right|>\lambda\left|I_{j}\right|$. Summing over $j$ and noting that almost every point of $E^{c}$ belongs to $C$ by the Lebesgue density theorem, we then get $\left|E^{c}\right|=\left|C \cap E^{c}\right|>\lambda|C|$. Thus $|C|<\left|E^{c}\right| / \lambda=(1-|E|) / \lambda<1$, and so $\left|C^{c}\right|>0$.

To finish the proof, note that for almost every $\zeta \in C^{c}$ we have $\zeta \in E$ and also $|I \cap E| \geq(1-\lambda)|I|=\frac{1}{2}|E||I|$ for all dyadic arcs $I$ containing $\zeta$. Moreover, for every arc $I(r \zeta) \subset \mathbb{T}$ there exists a dyadic arc $I$ such that $\zeta \in I \subset I(r \zeta)$ and $|I|>\frac{1}{4}|I(r \zeta)|$. These observations prove the lemma with $E^{\prime}=C^{c} \cap E$.

Proof of Theorem 2. As a preparatory step we first establish a Möbius-invariant version of condition (L). Let $\varphi_{b}=\varphi \circ \sigma_{b}$ for $b \in \mathbb{D}$. Then the following identity can be verified just by inspection and using the self-inverse property of the automorphisms:

$$
\sigma_{\varphi_{b}(a)} \circ \varphi_{b} \circ \sigma_{a}=\left[\sigma_{\varphi\left(\sigma_{b}(a)\right)} \circ \varphi \circ \sigma_{\sigma_{b}(a)}\right] \circ\left[\sigma_{\sigma_{b}(a)} \circ \sigma_{b} \circ \sigma_{a}\right] .
$$

Note that the composite mapping enclosed in the last brackets is a disc automorphism that fixes the origin, hence a rotation. Therefore

$$
\left\|\sigma_{\varphi_{b}(a)} \circ \varphi_{b} \circ \sigma_{a}\right\|_{H^{2}}=\left\|\sigma_{\varphi\left(\sigma_{b}(a)\right)} \circ \varphi \circ \sigma_{\sigma_{b}(a)}\right\|_{H^{2}} .
$$

Now, in view of (2.1) and the fact that $P_{a}(\zeta) \geq \frac{1}{4}|I(a)|^{-1}$ for $\zeta \in I(a)$, condition (L) implies the following: Given $\varepsilon>0$, there exists $\eta<1$ such that

$$
\frac{1}{|I(a)|} \int_{I(a)} \rho\left(\varphi_{b}(\zeta), \varphi_{b}(a)\right)^{2}|d \zeta| \leq \varepsilon
$$

whenever $a$ and $b$ satisfy $\left|\varphi_{b}(a)\right| \geq \eta$.

For the actual proof of Theorem 2 we argue by contradiction, assuming that (L) holds but (S2) does not. Since (S2) fails, there are constants $R<1$ and $c>0$, 
points $b_{k} \in \mathbb{D}$, and numbers $0<t_{k}<1$ with $t_{k} \rightarrow 1$ such that for all $k \geq 1$ we have $\left|\varphi\left(b_{k}\right)\right| \leq R$ and the sets

$$
E_{k}=\left\{\zeta \in \mathbb{T}: \text { the radial limit } \varphi_{k}(\zeta) \text { exists and }\left|\varphi_{k}(\zeta)\right|>t_{k}\right\}
$$

satisfy $\left|E_{k}\right| \geq c$, where $\varphi_{k}=\varphi \circ \sigma_{b_{k}}$. By Lemma 3 we can further find sets $E_{k}^{\prime} \subset E_{k}$ such that $\left|E_{k}^{\prime}\right|>0$ and

$$
\frac{\left|I(r \zeta) \cap E_{k}\right|}{|I(r \zeta)|} \geq \frac{c}{8} \quad \text { for } 0 \leq r<1, \zeta \in E_{k}^{\prime} .
$$

Let $\varepsilon=c / 16$. We may choose $\eta$ large enough so that $R<\eta<1$ and (2.2) holds for $\left|\varphi_{b}(a)\right| \geq \eta$. Fix $k$ such that $t_{k} \geq \eta$. Recall that by the definition of $E_{k}$ we have $\left|\varphi_{k}(r \zeta)\right| \rightarrow\left|\varphi_{k}(\zeta)\right|>t_{k}$ as $r \rightarrow 1$ for each $\zeta \in E_{k}$. In particular, we can fix a point $\zeta_{k} \in E_{k}^{\prime}$ with this property. Moreover, since $\left|\varphi_{k}(0)\right|=\left|\varphi\left(b_{k}\right)\right| \leq R$, it follows from continuity that there is a radius $0<r_{k}<1$ such that $\left|\varphi_{k}\left(r_{k} \zeta_{k}\right)\right|=\eta$. Let $a_{k}=r_{k} \zeta_{k}$. By elementary geometry it holds for each $\zeta \in E_{k}$ that $\rho\left(\varphi_{k}(\zeta), \varphi_{k}\left(a_{k}\right)\right) \geq \rho\left(t_{k}, \eta\right)$. Hence we can use (2.3) to obtain the estimate

$$
\frac{1}{\left|I\left(a_{k}\right)\right|} \int_{I\left(a_{k}\right)} \rho\left(\varphi_{k}(\zeta), \varphi_{k}\left(a_{k}\right)\right)^{2}|d \zeta| \geq \frac{\left|I\left(a_{k}\right) \cap E_{k}\right|}{\left|I\left(a_{k}\right)\right|} \rho\left(t_{k}, \eta\right)^{2} \geq \frac{c}{8} \rho\left(t_{k}, \eta\right)^{2} .
$$

Since this estimate holds for all sufficiently large $k$, we may let $k \rightarrow \infty$. In this case $\rho\left(t_{k}, \eta\right) \rightarrow 1$, which leads to a contradiction with (2.2) by the choice of $\varepsilon$.

Remark 4. It is appropriate to note that condition (S2) alone does not ensure the compactness of $C_{\varphi}$ on BMOA. For example, if $\varphi(z)=\frac{1}{2}(1+z)$, then one may check that (S2) holds but $C_{\varphi}$ fails to be compact. For instance, it is not difficult to see that $\left\|\sigma_{\varphi(a)} \circ \varphi \circ \sigma_{a}\right\|_{H^{2}} \rightarrow 1$ as $a \rightarrow 1$. We leave the details to the reader.

We close this section by addressing a question recently posed by Wulan, Zheng and Zhu [WZZ]. Based on an earlier work by Wulan [W], they showed that the single condition

$$
\lim _{n \rightarrow \infty}\left|\varphi^{n}\right|_{*}=0
$$

characterizes the compactness of $C_{\varphi}$ on $B M O A$. The earlier result in [W] involved the additional condition

$$
\lim _{|a| \rightarrow 1}\left|\sigma_{a} \circ \varphi\right|_{*}=0
$$

and so it was asked in [WZZ, Sec. 4] whether (W2) alone would suffice to characterize when $C_{\varphi}$ is compact on $B M O A$. This is indeed the case.

Corollary 5. Let $\varphi: \mathbb{D} \rightarrow \mathbb{D}$ be an analytic map. Then $C_{\varphi}$ is compact on BMOA if and only if (W2) holds.

Proof. It is enough to observe that $\left|\sigma_{\varphi(a)} \circ \varphi\right|_{*} \geq\left\|\sigma_{\varphi(a)} \circ \varphi \circ \sigma_{a}\right\|_{H^{2}}$, whence (W2) trivially implies $(\mathrm{L})$. 


\section{WEAK COMPACTNESS}

After the work of the preceding section the only step that remains to be proved in Theorem 1 is that (iii) implies (iv). Equivalently, if the map $\varphi$ fails to satisfy condition $(\mathbb{D})$, then we must show that the composition operator $C_{\varphi}$ is not weakly compact on $B M O A$. This will be accomplished separately in Proposition 8 below.

Our argument depends on the following proposition which is essentially due to Leǐbov [Le] and provides information about the subspace structure of VMOA (cf. Remark 7 below). As usual, here $c_{0}$ denotes the Banach space of complex sequences converging to zero endowed with the supremum norm $\|\cdot\|_{\infty}$. The proof given below is an adaptation of Lerbov's argument; he worked in the space $V M O(\mathbb{T})$ on the unit circle, but we work directly in the disc.

Proposition 6. Let $\left(f_{n}\right)$ be a sequence in VMOA such that $\left\|f_{n}\right\|_{*}=1$ for all $n$ and $\left\|f_{n}\right\|_{H^{2}} \rightarrow 0$ as $n \rightarrow \infty$. Then there exists a subsequence $\left(f_{n_{k}}\right)$ which is equivalent to the natural basis of $c_{0}$; that is, for which the map $\left(\lambda_{k}\right) \mapsto \sum_{k} \lambda_{k} f_{n_{k}}$ is an isomorphism from $c_{0}$ into $V M O A$.

Proof. For brevity we write

$$
\gamma(f, a)=\left\|f \circ \sigma_{a}-f(a)\right\|_{H^{2}}
$$

whenever $f \in H^{2}$ and $a \in \mathbb{D}$. Note that $\gamma(f, a)$ defines a seminorm with respect to $f$ for each $a$. We also have $\gamma(f, a) \leq\left\|f \circ \sigma_{a}\right\|_{H^{2}} \leq c_{a}\|f\|_{H^{2}}$ for some $c_{a}>0$, where $c_{a}$ is an increasing function of $|a|$. Therefore

$$
\sup \left\{\gamma\left(f_{n}, a\right):|a| \leq r\right\} \rightarrow 0 \quad \text { as } n \rightarrow \infty
$$

for any $0<r<1$. On the other hand, the $V M O A$ condition says that $\gamma\left(f_{n}, a\right) \rightarrow 0$ as $|a| \rightarrow 1$ for each $n$. Proceeding inductively, we can use these properties of $\left(f_{n}\right)$ to find increasing sequences of integers $n_{k} \geq 1$ and numbers $0<r_{k}<1$ (starting with $r_{1}=\frac{1}{2}$, say) such that for each $k \geq 1$ one has $\left\|f_{n_{k}}\right\|_{H^{2}}<2^{-k-1}$ and

$$
\sup _{|a| \leq r_{k}} \gamma\left(f_{n_{k}}, a\right)<2^{-k-1}, \quad \sup _{|a| \geq r_{k+1}} \gamma\left(f_{n_{k}}, a\right)<2^{-k-1} .
$$

For every $a \in \mathbb{D}$ we then have $\gamma\left(f_{n_{k}}, a\right)<2^{-k-1}$ for all except possibly one index $k$, for which $\gamma\left(f_{n_{k}}, a\right) \leq 1$. Hence $\sum_{k} \gamma\left(f_{n_{k}}, a\right)<1+\frac{1}{2}=\frac{3}{2}$.

Given a sequence $\lambda=\left(\lambda_{k}\right) \in c_{0}$, define

$$
S \lambda=\sum_{k=1}^{\infty} \lambda_{k} f_{n_{k}} .
$$

The exponential decay of the $H^{2}$ norms of the functions $f_{n_{k}}$ ensures that the series converges in $H^{2}$ and hence pointwise. In particular, from the fact that $\left|f_{n_{k}}(0)\right| \leq$ $\left\|f_{n_{k}}\right\|_{H^{2}}<2^{-k-1}$ we get that $|S \lambda(0)| \leq \frac{1}{2}\|\lambda\|_{\infty}$. In addition, for $a \in \mathbb{D}$,

$$
\gamma(S \lambda, a) \leq \sum_{k=1}^{\infty}\left|\lambda_{k}\right| \gamma\left(f_{n_{k}}, a\right) \leq \frac{3}{2}\|\lambda\|_{\infty} .
$$


Hence $\|S \lambda\|_{*} \leq 2\|\lambda\|_{\infty}$. To check that $S \lambda \in V M O A$, we let $\varepsilon>0$ and choose an integer $K$ such that $\left|\lambda_{k}\right| \leq \varepsilon$ for $k>K$. Then, by estimating as above we have

$$
\gamma(S \lambda, a) \leq\|\lambda\|_{\infty} \sum_{k=1}^{K} \gamma\left(f_{n_{k}}, a\right)+\frac{3}{2} \varepsilon .
$$

Since $\gamma\left(f_{n_{k}}, a\right) \rightarrow 0$ as $|a| \rightarrow 1$ for each $k$, and $\varepsilon>0$ was arbitrary, this implies that $S \lambda \in V M O A$. Thus we have proved that $S$ is a bounded linear operator from $c_{0}$ into VMOA.

It remains to show that $S$ is bounded below. Given $\lambda=\left(\lambda_{k}\right) \in c_{0}$, we first choose an index $K$ for which $\left|\lambda_{K}\right|=\|\lambda\|_{\infty}$. Then we pick a point $a \in \mathbb{D}$ such that $\gamma\left(f_{n_{K}}, a\right) \geq \frac{3}{4}$; this is possible since $\left\|f_{n_{K}}\right\|_{*}=1$ and $\left|f_{n_{K}}(0)\right|<\frac{1}{4}$. Note that for $k \neq K$ we necessarily have $\gamma\left(f_{n_{k}}, a\right)<2^{-k-1}$. Therefore, by employing the triangle inequality we get that

$$
\begin{aligned}
\|S \lambda\|_{*} & \geq \gamma(S \lambda, a) \geq\left|\lambda_{K}\right| \gamma\left(f_{n_{K}}, a\right)-\sum_{k \neq K}\left|\lambda_{k}\right| \gamma\left(f_{n_{k}}, a\right) \\
& \geq \frac{3}{4}\|\lambda\|_{\infty}-\frac{1}{2}\|\lambda\|_{\infty}=\frac{1}{4}\|\lambda\|_{\infty} .
\end{aligned}
$$

Remark 7. Let $X$ be a closed subspace of $V M O A$. As a consequence of Proposition 6 one has the following dichotomy (see [Le]): either $X$ contains an isomorphic copy of $c_{0}$ or the natural embedding of $X$ into $H^{2}$ is an isomorphism. An analogous result in the setting of martingale $V M O$ spaces has been proved in [MS]. We point out here that the subspace structure of $B M O A$ is very complicated; see $[\mathrm{M}]$.

As noted at the beginning of the present section, the following proposition completes the proof of Theorem 1 .

Proposition 8. Let $\varphi: \mathbb{D} \rightarrow \mathbb{D}$ be an analytic map and suppose that condition (L) fails. Then the composition operator $C_{\varphi}: B M O A \rightarrow B M O A$ fixes a copy of $c_{0}$ and therefore it is not weakly compact.

Proof. Since $(\mathbb{L})$ fails to hold, we can find points $a_{n} \in \mathbb{D}$ such that $\left|\varphi\left(a_{n}\right)\right| \rightarrow 1$ and

$$
\left\|\sigma_{\varphi\left(a_{n}\right)} \circ \varphi \circ \sigma_{a_{n}}\right\|_{H^{2}} \geq c
$$

for some $c>0$. Put $f_{n}=\sigma_{\varphi\left(a_{n}\right)}-\varphi\left(a_{n}\right)$. Then $f_{n}(0)=0$ and, for each $a \in \mathbb{D}$,

$$
\left\|f_{n} \circ \sigma_{a}-f_{n}(a)\right\|_{H^{2}}=\left\|\sigma_{\varphi\left(a_{n}\right)} \circ \sigma_{a}-\sigma_{\varphi\left(a_{n}\right)}(a)\right\|_{H^{2}}=\sqrt{1-\left|\sigma_{\varphi\left(a_{n}\right)}(a)\right|^{2}} .
$$

The last equality can be seen by using the fact that $\sigma_{\varphi\left(a_{n}\right)} \circ \sigma_{a}$ is an inner function. Now it follows easily that $f_{n} \in V M O A$ and $\left\|f_{n}\right\|_{*}=1$ for each $n$. By taking $a=0$ we obtain that $\left\|f_{n}\right\|_{H^{2}} \rightarrow 0$ as $n \rightarrow \infty$. Moreover,

$$
\left\|C_{\varphi} f_{n}\right\|_{*} \geq\left\|f_{n} \circ \varphi \circ \sigma_{a_{n}}-f_{n}\left(\varphi\left(a_{n}\right)\right)\right\|_{H^{2}}=\left\|\sigma_{\varphi\left(a_{n}\right)} \circ \varphi \circ \sigma_{a_{n}}\right\|_{H^{2}} \geq c .
$$

According to Proposition 6 there is a subsequence $\left(f_{n_{k}}\right)$ which is equivalent to the natural basis of $c_{0}$. In particular, $\left(C_{\varphi} f_{n_{k}}\right)$ is a weak-null sequence in BMOA. By applying the Bessaga-Pełczyński selection principle (see e.g. [AK, 1.3.10]) to $\left(C_{\varphi} f_{n_{k}}\right)$ 
we can pass to a further subsequence, still denoted $\left(f_{n_{k}}\right)$, such that $\left(C_{\varphi} f_{n_{k}}\right)$ is a seminormalized basic sequence in $B M O A$. It follows that there are constants $A, B>0$ so that

$$
A \cdot\|\lambda\|_{\infty} \leq\left\|\sum_{k} \lambda_{k} C_{\varphi} f_{n_{k}}\right\|_{*} \leq\left\|C_{\varphi}\right\| \cdot\left\|\sum_{k} \lambda_{k} f_{n_{k}}\right\|_{*} \leq B \cdot\left\|C_{\varphi}\right\|\|\lambda\|_{\infty}
$$

holds for any sequence $\lambda=\left(\lambda_{k}\right) \in c_{0}$. (To find $A$ just apply the biorthogonal basis functionals to $\sum_{k} \lambda_{k} C_{\varphi} f_{n_{k}}$.) These estimates state that the restriction of $C_{\varphi}$ to the closed subspace of BMOA spanned by the sequence $\left(f_{n_{k}}\right)$ is an isomorphism on a linearly isomorphic copy of $c_{0}$, and we are done.

Remark 9. (1) Theorem 1 and its condition $(\mathbb{L})$ also characterize the compactness, as well as the weak compactness, of $C_{\varphi}$ on the space $B M O$ identified with the space of harmonic functions $\mathbb{D} \rightarrow \mathbb{C}$ whose boundary values have bounded mean oscillation. Indeed, it is known that a composition operator is compact on $B M O A$ if and only if it is compact on $B M O$ (see e.g. $\mathrm{BCM}$, Thm 3.5]). Hence it remains to observe that if $C_{\varphi}$ is weakly compact on $B M O$, then it is weakly compact on the subspace $B M O A$ as well so that $(\mathrm{L})$ holds.

(2) Theorem 1 1 allows one to complete some characterizations in [L1, L2] as follows: if $X$ is an infinite-dimensional complex reflexive Banach space, then $C_{\varphi}$ is weakly compact on certain $X$-valued versions of $B M O A$ precisely when $C_{\varphi}$ is compact on $B M O A$. We refer to [L1, L2] for a description of this setting.

\section{A CONDition on MEAN Oscillation}

In this section our aim is to examine the function-theoretic meaning of condition (L) by revisiting the point of view that we already touched upon in Section 2, That is, (L) can be thought of as a kind of pseudo-hyperbolic vanishing mean oscillation condition for the boundary values of $\varphi$ over certain arcs in $\mathbb{T}$; see Proposition 10 below.

To begin with we introduce some notation. When $\varphi: \mathbb{D} \rightarrow \mathbb{D}$ is an analytic map and $I$ is an $\operatorname{arc}$ of $\mathbb{T}$, denote

$$
\varphi_{I}=\frac{1}{|I|} \int_{I} \varphi=\frac{1}{|I|} \int_{I} \varphi(\zeta)|d \zeta|
$$

for the integral average of $\varphi$ over $I$. Here and elsewhere in this section all integrals over subsets of $\mathbb{T}$ are calculated with respect to the normalized Lebesgue arc-length measure. Also recall from Section 2 that $I\left(r e^{i \theta}\right)=\left\{e^{i t}:|t-\theta| \leq \pi(1-r)\right\}$ for $r e^{i \theta} \in \mathbb{D}$.

Proposition 10. For any analytic map $\varphi: \mathbb{D} \rightarrow \mathbb{D}$ condition $(\mathbb{L})$ is equivalent to the following:

$$
\frac{1}{|I|^{2}} \int_{I} \int_{I} \rho(\varphi(\zeta), \varphi(\xi))^{2}|d \zeta||d \xi| \rightarrow 0 \quad \text { as }\left|\varphi_{I}\right| \rightarrow 1,
$$

where $I \subset \mathbb{T}$ are arcs.

In the proof of this proposition we will make use of the following easy estimate for the Poisson kernel, whose verification we leave to the reader: for every $a \in \mathbb{D}$,

$$
\frac{1}{4|I(a)|} \leq P_{a}(\zeta) \leq \frac{2}{|I(a)|}, \quad \zeta \in I(a) .
$$


We next record a simple auxiliary result, which isolates a crucial step in proving Proposition 10

Lemma 11. For $a \in \mathbb{D}$ we have $|\varphi(a)| \rightarrow 1$ if and only if $\left|\varphi_{I(a)}\right| \rightarrow 1$.

Proof. The left-to-right implication is easy to prove. In fact, assuming that $\varphi(a) \geq 0$ (as we may, after applying a rotation), we get by using (4.1) that

$$
1-|\varphi(a)|=\int_{\mathbb{T}}(1-\operatorname{Re} \varphi) P_{a} \geq \frac{1}{4|I(a)|} \int_{I(a)}(1-\operatorname{Re} \varphi) \geq \frac{1}{4}\left(1-\left|\varphi_{I(a)}\right|\right) .
$$

This clearly shows that $|\varphi(a)| \rightarrow 1$ implies $\left|\varphi_{I(a)}\right| \rightarrow 1$.

For the reverse implication, we may assume that $\varphi_{I(a)} \geq 1-\delta$ for some $0<\delta<\frac{1}{2}$. Let $E=\{\zeta \in I(a): \operatorname{Re} \varphi(\zeta) \geq 1-2 \delta\}$. Since $\operatorname{Re} \varphi \leq 1$, we must have $|E| \geq \frac{1}{2}|I(a)|$. Consider the positive harmonic function $u=\log (2 /|1-\varphi|)$. It is geometrically obvious that $|1-\varphi| \leq c \sqrt{\delta}$ on $E$ for some constant $c>0$. Hence

$$
u(a) \geq \int_{\mathbb{T}} u P_{a} \geq\left(\log \frac{2}{c \sqrt{\delta}}\right) \int_{E} P_{a} \geq \frac{1}{8}\left(\log \frac{2}{c \sqrt{\delta}}\right) .
$$

Since $|1-\varphi(a)|=2 e^{-u(a)}$, we deduce from this estimate that $1-|\varphi(a)| \leq|1-\varphi(a)| \rightarrow 0$ as $\delta \rightarrow 0$.

Proof of Proposition 10. We start by proving the necessity of (A). By the preceding lemma $\left|\varphi_{I}\right| \rightarrow 1$ implies that $\left|\varphi\left(a_{I}\right)\right| \rightarrow 1$. Hence (2.1) and the left-hand side of (4.1) yield

$$
\frac{1}{|I|} \int_{\mathbb{T}} \rho\left(\varphi(\zeta), \varphi\left(a_{I}\right)\right)^{2}|d \zeta| \rightarrow 0 \quad \text { as }\left|\varphi_{I}\right| \rightarrow 1,
$$

where $I \subset \mathbb{T}$ is an arc and $a_{I} \in \mathbb{D}$ is the unique point for which $I=I\left(a_{I}\right)$. Then (A) is obtained from (A') by a simple application of the triangle inequality $\rho(\varphi(\zeta), \varphi(\xi)) \leq$ $\rho\left(\varphi(\zeta), \varphi\left(a_{I}\right)\right)+\rho\left(\varphi(\xi), \varphi\left(a_{I}\right)\right)$.

To prove the sufficiency of (A) we will show that

$$
J(a)=\int_{\mathbb{T}} \int_{\mathbb{T}} \rho(\varphi(\zeta), \varphi(\xi))^{2} P_{a}(\zeta) P_{a}(\xi)|d \zeta||d \xi| \rightarrow 0 \quad \text { as }|\varphi(a)| \rightarrow 1 .
$$

In view of (2.1) this actually implies $(\mathbb{L})$, because the function $w \mapsto \rho(z, w)^{2}$ is subharmonic in $\mathbb{D}$ and therefore $\int_{\mathbb{T}} \rho(z, \varphi(\xi))^{2} P_{a}(\xi)|d \xi| \geq \rho(z, \varphi(a))^{2}$ for every $z \in \overline{\mathbb{D}}$.

Let $\varepsilon>0$. For each $a \in \mathbb{D}$ we can choose a point $a^{\prime}$ on the line segment between 0 and $a$ such that $\int_{I\left(a^{\prime}\right)} P_{a} \geq 1-\varepsilon$ and $1-\left|a^{\prime}\right| \leq c_{\varepsilon}(1-|a|)$ for some constant $c_{\varepsilon}>0$. For real $a$ close to 1 this can be seen by integrating the estimate $P_{a}\left(e^{i t}\right) \geq$ $\left(1-a^{2}\right) /\left[(1-a)^{2}+t^{2}\right]$ over an interval $|t| \leq c(1-a)$ and letting $c \rightarrow \infty$. Now $\int_{\mathbb{T} \backslash I\left(a^{\prime}\right)} P_{a} \leq \varepsilon$, and since $\rho \leq 1$, we can estimate

$$
\begin{aligned}
J(a) & \leq 2 \varepsilon+\int_{I\left(a^{\prime}\right)} \int_{I\left(a^{\prime}\right)} \rho(\varphi(\zeta), \varphi(\xi))^{2} P_{a}(\zeta) P_{a}(\xi)|d \zeta||d \xi| \\
& \leq 2 \varepsilon+\frac{4 c_{\varepsilon}^{2}}{\left|I\left(a^{\prime}\right)\right|^{2}} \int_{I\left(a^{\prime}\right)} \int_{I\left(a^{\prime}\right)} \rho(\varphi(\zeta), \varphi(\xi))^{2}|d \zeta||d \xi|
\end{aligned}
$$

by using the right-hand side of (4.1) in the last step. According to the Schwarz-Pick inequality we have $\rho\left(\varphi(a), \varphi\left(a^{\prime}\right)\right) \leq \rho\left(a, a^{\prime}\right) \leq c_{\varepsilon}^{\prime}$ for some $c_{\varepsilon}^{\prime}<1$ due to the fact that $1-\left|a^{\prime}\right| \leq c_{\varepsilon}(1-|a|)$. Thus $|\varphi(a)| \rightarrow 1$ implies that $\left|\varphi\left(a^{\prime}\right)\right| \rightarrow 1$, which, in turn, yields 
$\left|\varphi_{I\left(a^{\prime}\right)}\right| \rightarrow 1$ by Lemma 11. By applying (A) to the arcs $I\left(a^{\prime}\right)$ we then deduce from the above estimate that $\lim \sup J(a) \leq 2 \varepsilon$ as $|\varphi(a)| \rightarrow 1$. Since $\varepsilon>0$ was arbitrary, this proves (4.2).

We summarize the principal function-theoretic compactness criteria for $C_{\varphi}$ on $B M O A$ in the following theorem. Criteria of a different nature are given in [BCM] and $[\mathrm{WX}$.

Theorem 12. Compactness and weak compactness of $C_{\varphi}: B M O A \rightarrow B M O A$ are equivalent to each of the conditions (S1), (L1), (W1), (W2), (स) and (A').

\section{Results FOR VMOA}

In this section we discuss the case where $\varphi \in V M O A$. Here simplified compactness criteria are available and new phenomena occur. Recall first that if $\varphi \in V M O A$ then $C_{\varphi}$ takes $V M O A$ into itself and $C_{\varphi}: B M O A \rightarrow B M O A$ can be identified with the biadjoint of its restriction to $V M O A$; see [CM. p. 939].

Let $\tau$ denote the hyperbolic metric in the unit disc, that is,

$$
\tau(z, w)=\frac{1}{2} \log \frac{1+\rho(z, w)}{1-\rho(z, w)},
$$

where $\rho(z, w)$ is the pseudo-hyperbolic distance between $z$ and $w$ (see e.g. [Z, Sec. 4.3]). Contrary to the pseudo-hyperbolic metric, $\tau$ is unbounded in $\mathbb{D}$ and it is appropriate to define $\tau(z, w)=\infty$ if $z$ and $w$ are distinct points (at least) one of which lies on the boundary.

We collect the main results in the case of $V M O A$ as follows.

Theorem 13. Let $\varphi: \mathbb{D} \rightarrow \mathbb{D}$ be an analytic map such that $\varphi \in V M O A$. Then the following conditions are equivalent:

(i) $C_{\varphi}: V M O A \rightarrow V M O A$ is compact.

(ii) $C_{\varphi}: V M O A \rightarrow V M O A$ is weakly compact.

(iii) $\lim _{|a| \rightarrow 1}\left\|\sigma_{\varphi(a)} \circ \varphi \circ \sigma_{a}\right\|_{H^{2}}=0$.

(iv) $\lim _{|a| \rightarrow 1} \int_{\mathbb{T}} \rho\left(\varphi\left(\sigma_{a}(\zeta)\right), \varphi(a)\right)^{2}|d \zeta|=0$.

(v) $\lim _{|I| \rightarrow 0} \frac{1}{|I|^{2}} \int_{I} \int_{I} \rho(\varphi(\zeta), \varphi(\xi))^{2}|d \zeta||d \xi|=0$, where $I \subset \mathbb{T}$ are arcs.

Further, (iv) and (ब्V) are equivalent to the following conditions involving the hyperbolic metric:

(iv') $\lim _{|a| \rightarrow 1} \int_{\mathbb{T}} \tau\left(\varphi\left(\sigma_{a}(\zeta)\right), \varphi(a)\right)|d \zeta|=0$.

(v') $\lim _{|I| \rightarrow 0} \frac{1}{|I|^{2}} \int_{I} \int_{I} \tau(\varphi(\zeta), \varphi(\xi))|d \zeta||d \xi|=0$, where $I \subset \mathbb{T}$ are arcs.

The main novelty of Theorem [13, as compared to Theorem 11, lies in conditions (iv') and ( $\overrightarrow{\mathrm{v}^{\prime}}$ ), which relate to vanishing mean oscillation with respect to the genuine hyperbolic metric. This also ties to earlier research on composition operators from the Bloch space to VMOA. Before embarking on the proof of Theorem 13 we discuss the interpretation of (iv') from the literature and draw some consequences. 
First note that if the integral $\int_{\mathbb{T}} \tau\left(\varphi\left(\sigma_{a}(\zeta)\right), \varphi(a)\right)|d \zeta|$ is finite for some $a \in \mathbb{D}$, then $|\varphi|<1$ a.e. on $\mathbb{T}$. Moreover, the integral stays bounded as $a$ varies on a compact subset of $\mathbb{D}$. Hence (iv') implies

$$
\sup _{a \in \mathbb{D}} \int_{\mathbb{T}} \tau\left(\varphi\left(\sigma_{a}(\zeta)\right), \varphi(a)\right)|d \zeta|<\infty,
$$

saying that $\varphi$ belongs to the hyperbolic BMOA class introduced by Yamashita $[\mathrm{Y}]$. Actually the fact that (iv) implies the finiteness of the integral in (5.1) for some $a \in \mathbb{D}$ is already non-trivial.

Recall that the Bloch space $\mathcal{B}$ consists of the analytic functions $f: \mathbb{D} \rightarrow \mathbb{C}$ for which $\sup _{z \in \mathbb{D}}\left|f^{\prime}(z)\right|\left(1-|z|^{2}\right)<\infty$. Then $\mathcal{B}$ becomes a Banach space equipped with the norm $|f(0)|+\sup _{z \in \mathbb{D}}\left|f^{\prime}(z)\right|\left(1-|z|^{2}\right)$. Composition operators $C_{\varphi}$ acting from $\mathcal{B}$ into VMOA or BMOA have been studied in e.g. [T], [CRU, [SZ, [MT, $[\mathrm{X}]$ and [LMT]. As observed by Makhmutov and Tjani [MT], it follows from the results of Choe, Ramey and Ullrich [CRU] combined with $[\mathrm{Y}]$ that $C_{\varphi}$ is bounded from $\mathcal{B}$ into $B M O A$ if and only if (5.1) holds. In addition, it was proved in [MT, Thm 6.1] that $C_{\varphi}$ is compact from $\mathcal{B}$ into VMOA if and only if (iv') holds. Therefore Theorem 13 has the following surprising consequence.

Corollary 14. Let $\varphi: \mathbb{D} \rightarrow \mathbb{D}$ be an analytic map with $\varphi \in V M O A$. Then $C_{\varphi}$ is compact VMOA $\rightarrow$ VMOA if and only if it is compact $\mathcal{B} \rightarrow$ VMOA.

This result was known earlier in the special case of boundedly valent symbols $\varphi$ whose image $\varphi(\mathbb{D})$ is contained in a polygon inscribed in $\overline{\mathbb{D}}$; see [MT, Thm 5.3]. Of course, in Corollary 14 the implication from right to left follows from the fact that $V M O A$ is continuously embedded in $\mathcal{B}$. Furthermore, it is relevant to note that $C_{\varphi}$ is bounded $\mathcal{B} \rightarrow V M O A$ if and only if it is compact $\mathcal{B} \rightarrow V M O A$; see [SZ, Thm 1.6].

Towards the proof of Theorem 13 we make some preliminary remarks. It was already observed by the first author [L3, Thm 4.3] that condition (iii) alone characterizes the compactness of $C_{\varphi}: V M O A \rightarrow V M O A$. At first sight (iii) might seem stronger than (L) because $|\varphi(a)| \rightarrow 1$ always implies $|a| \rightarrow 1$ by the Schwarz lemma. For the reader's convenience we include a direct function-theoretic argument proving the equivalence of these two conditions for symbols $\varphi \in V M O A$.

Lemma 15. Let $\varphi: \mathbb{D} \rightarrow \mathbb{D}$ be an analytic map. Then condition (iii) of Theorem 13 holds if and only if $\varphi \in V M O A$ and $(\mathrm{L})$ holds.

Proof. Let $\varphi_{a}=\sigma_{\varphi(a)} \circ \varphi \circ \sigma_{a}$. By the self-inverse property of $\sigma_{\varphi(a)}$ we may write $\varphi \circ \sigma_{a}=\sigma_{\varphi(a)} \circ \varphi_{a}$, from which it follows that

$$
\left|\left(\varphi \circ \sigma_{a}\right)(z)-\varphi(a)\right|=\frac{1-|\varphi(a)|^{2}}{\left|1-\overline{\varphi(a)} \varphi_{a}(z)\right|}\left|\varphi_{a}(z)\right| .
$$

This yields $\left\|\varphi \circ \sigma_{a}-\varphi(a)\right\|_{H^{2}} \leq 2\left\|\varphi_{a}\right\|_{H^{2}}$. Hence (iii) implies that $\varphi \in V M O A$.

Conversely note that if $(\mathrm{L})$ holds but (iiii) fails, then there exists a sequence $\left(a_{n}\right)$ such that $\left|a_{n}\right| \rightarrow 1$ while $\left|\varphi\left(a_{n}\right)\right| \leq r<1$ and $\left\|\varphi_{a_{n}}\right\|_{H^{2}} \geq c>0$ for all $n$. Then (5.2) implies that $\left\|\varphi \circ \sigma_{a_{n}}-\varphi\left(a_{n}\right)\right\|_{H^{2}} \geq(1-r)\left\|\varphi_{a_{n}}\right\|_{H^{2}} \geq(1-r) c$, whence $\varphi \notin V M O A$. This proves the lemma. 
Proof of Theorem [13. Recall that the operator $C_{\varphi}: B M O A \rightarrow B M O A$ is the biadjoint of the restriction $C_{\varphi}: V M O A \rightarrow V M O A$, since here $\varphi \in V M O A$. Hence, according to Theorem 1, conditions (ii) and (iii) are both equivalent to (). On the other hand, in this case ( $(\mathrm{L})$ and (iii) are equivalent by Lemma 15 . We refer to Remark 18 below for an approach to the equivalences between conditions (ii)-(iii) which does not depend on Section 2 ,

Conditions (iii) and (iv) are restatements of each other according to (2.1). Furthermore, the equivalence of (iii) and (iv) is proved in the same way as Proposition 10 , instead of invoking Lemma 11] we just observe that for points $a \in \mathbb{D}$ one has $|a| \rightarrow 1$ if and only if $|I(a)| \rightarrow 0$.

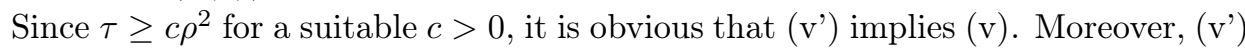
can be deduced from (iv') by making a change of variable, using the lower estimate from (4.1) for the Poisson kernel and applying the triangle inequality as in the first part of the proof of Proposition 10. The crucial remaining step in the proof of Theorem 13 consists of verifying the implication that the pseudo-hyperbolic condition (iv) implies the hyperbolic condition (iv'). We isolate this more technical result below, which then completes the proof of the theorem.

Proposition 16. Let $\varphi: \mathbb{D} \rightarrow \mathbb{D}$ be an analytic map. Then condition (iv) implies condition (iv') in Theorem 13.

The argument will employ ideas of Wik Wi] related to his elementary approach to the John-Nirenberg inequality for $B M O$ functions. In particular, we will require the following one-dimensional special case of [Wi, Lemma 1]:

Lemma 17. Suppose that $0<\lambda<1$ and $E \subset[0,1]$ is any measurable set having Lebesgue measure $|E| \leq \lambda$. Then there is a sequence $Q_{1}, Q_{2}, \ldots$ of closed dyadic intervals of $[0,1]$, having pairwise disjoint interiors, such that $\frac{1}{2} \lambda\left|Q_{k}\right| \leq\left|Q_{k} \cap E\right| \leq$ $\lambda\left|Q_{k}\right|$ for $k \geq 1$ and $\left|E \backslash \bigcup_{k} Q_{k}\right|=0$.

Proof of Proposition [16, Assuming that condition (iv) (and equivalently also (v)) holds, we split the proof into two steps. As the first step we show:

Claim 1. $\lim _{|a| \rightarrow 1} \frac{1}{|I(a)|} \int_{I(a)} \tau(\varphi(\zeta), \varphi(a))|d \zeta|=0$.

To begin recall from Section 2 that condition (iv) implies that $|\varphi|<1$ a.e. on $\mathbb{T}$ (this fact can alternatively be deduced by observing that (ii) implies the compactness of $C_{\varphi}$ on $H^{2}$ by [BCM, Thm 4.1]). Towards the proof of Claim 1 we first deduce from (iv) by a change of variable and (4.1) that

$$
\lim _{|a| \rightarrow 1} \frac{1}{|I(a)|} \int_{I(a)} \rho(\varphi(\zeta), \varphi(a))^{2}|d \zeta|=0,
$$

where $I(a)=\left\{e^{i t}:|t-\theta| \leq \pi(1-r)\right\}$ is the subarc of $\mathbb{T}$ associated to $a=r e^{i \theta} \in \mathbb{D}$. Hence we may pick $\delta>0$ small enough so that

$$
\frac{1}{|I(a)|} \int_{I(a)} \rho(\varphi(\zeta), \varphi(a))^{2}|d \zeta|<\frac{1}{4}
$$

whenever $a \in \mathbb{D}$ satisfies $|a|>1-\delta$. 
Let $\varepsilon \in(0,1 / 32)$. According to (v) we may decrease $\delta>0$, if necessary, to ensure that for all $a \in \mathbb{D}$ with $|a|>1-\delta$ we also have

$$
\frac{1}{|I(a)|^{2}} \int_{I(a)} \int_{I(a)} \rho(\varphi(\zeta), \varphi(\xi))^{2}|d \zeta \| d \xi|<\varepsilon .
$$

Fix such a point $a$ and put

$$
C_{k}=\{\zeta \in I(a): \tau(\varphi(\zeta), \varphi(a)) \geq k\}, \quad k=0,1,2, \ldots,
$$

whence $I(a)=C_{0} \supset C_{1} \supset C_{2} \supset \cdots$. Observe that if $\zeta \in C_{1}$, then the definition of the hyperbolic metric yields $\rho(\varphi(\zeta), \varphi(a)) \geq \beta$, where $\beta=\frac{e^{2}-1}{e^{2}+1}>1 / \sqrt{2}$. One gets from (5.4) that

$$
\beta^{2} \frac{\left|C_{1}\right|}{|I(a)|} \leq \frac{1}{|I(a)|} \int_{I(a)} \rho(\varphi(\zeta), \varphi(a))^{2}|d \zeta|<\frac{1}{4},
$$

whence $\left|C_{1}\right| \leq \frac{1}{2}|I(a)|$.

Let $k \geq 1$ be fixed. Then we may apply Lemma 17 to the set $C_{k}$ relative to the $\operatorname{arc} I(a)$ with $\lambda=\frac{1}{2}$, which gives a sequence $J_{1}, J_{2}, \ldots$ of subarcs of $I(a)$ with disjoint interiors such that for each $\ell \geq 1$

$$
\left|C_{k} \cap J_{\ell}\right| \geq \frac{1}{4}\left|J_{\ell}\right|, \quad\left|C_{k}^{c} \cap J_{\ell}\right| \geq \frac{1}{2}\left|J_{\ell}\right|
$$

and

$$
\left|C_{k} \backslash \bigcup_{\ell=1}^{\infty} J_{\ell}\right|=0
$$

Observe next that if $\zeta \in C_{k}^{c}$ and $\xi \in C_{k+1}$, then $\tau(\varphi(\zeta), \varphi(\xi)) \geq \tau(\varphi(\xi), \varphi(a))-$ $\tau(\varphi(\zeta), \varphi(a)) \geq 1$, so that $\rho(\varphi(\zeta), \varphi(\xi))^{2} \geq \beta^{2}>0$. Consequently we get from (5.5), (5.6) and the assumption on $a$ that

$$
\begin{aligned}
\varepsilon & >\frac{1}{\left|J_{\ell}\right|^{2}} \int_{J_{\ell}} \int_{J_{\ell}} \rho(\varphi(\zeta), \varphi(\xi))^{2}|d \zeta||d \xi| \\
& \geq \beta^{2} \frac{\left|C_{k}^{c} \cap J_{\ell}\right|}{\left|J_{\ell}\right|} \cdot \frac{\left|C_{k+1} \cap J_{\ell}\right|}{\left|J_{\ell}\right|} \geq \frac{1}{4} \frac{\left|C_{k+1} \cap J_{\ell}\right|}{\left|J_{\ell}\right|} .
\end{aligned}
$$

Thus $\left|C_{k+1} \cap J_{\ell}\right| \leq 4 \varepsilon\left|J_{\ell}\right|$ for $\ell \geq 1$. We sum this inequality over $\ell$ and employ (5.6) and (5.7) together with the essential disjointness of the subarcs $J_{\ell}$ to obtain

$$
\left|C_{k+1}\right|=\sum_{\ell=1}^{\infty}\left|C_{k+1} \cap J_{\ell}\right| \leq 4 \varepsilon \sum_{\ell=1}^{\infty}\left|J_{\ell}\right| \leq 16 \varepsilon \sum_{\ell=1}^{\infty}\left|C_{k} \cap J_{\ell}\right|=16 \varepsilon\left|C_{k}\right| .
$$

In particular, since $\varepsilon<1 / 32$, we get by induction that $\left|C_{k}\right| \leq 2^{2-k}\left|C_{2}\right|$ for $k \geq 2$.

Note that $k \leq \tau(\varphi(\zeta), \varphi(a))<k+1$ whenever $\zeta \in C_{k} \backslash C_{k+1}$ and $k \geq 0$. Employing the short-hand notation $\{\tau<2\}$ for the set $\{\zeta \in I(a): \tau(\varphi(\zeta), \varphi(a))<2\}=C_{0} \backslash C_{2}$ we thus get that

$$
\begin{aligned}
\int_{I(a)} \tau(\varphi(\zeta), \varphi(a))|d \zeta| & =\int_{\{\tau<2\}} \tau(\varphi(\zeta), \varphi(a))|d \zeta|+\sum_{k=2}^{\infty} \int_{C_{k} \backslash C_{k+1}} \tau(\varphi(\zeta), \varphi(a))|d \zeta| \\
& \leq \int_{\{\tau<2\}} \tau(\varphi(\zeta), \varphi(a))|d \zeta|+\sum_{k=2}^{\infty}(k+1)\left|C_{k}\right| .
\end{aligned}
$$


After division by $|I(a)|$ the last term is less than $\left|C_{2}\right||I(a)|^{-1} \sum_{k=2}^{\infty}(k+1) 2^{2-k} \leq$ $128 \varepsilon$, which tends to 0 as $\varepsilon \rightarrow 0$. On the other hand, in the set $\{\tau<2\}$ we have $\tau(\varphi(\zeta), \varphi(a)) \leq c \rho(\varphi(\zeta), \varphi(a))^{2}$ with a universal constant $c>0$, so that also

$$
\lim _{|a| \rightarrow 1} \frac{1}{|I(a)|} \int_{\{\tau<2\}} \tau(\varphi(\zeta), \varphi(a))|d \zeta|=0
$$

in view of (5.3). This finishes the proof of Claim 1

As the final step we show that the condition of Claim 1 implies the desired hyperbolic condition (iv') of Theorem 13 The required argument is quite standard but more technical than the analogous fact for the pseudo-hyperbolic distance $\rho$ in Section 4 because the hyperbolic distance $\tau$ is unbounded. We omit some computational details.

Claim 2. $\int_{\mathbb{T}} \tau\left(\varphi\left(\sigma_{a}(\zeta)\right), \varphi(a)\right)|d \zeta|=\int_{\mathbb{T}} \tau(\varphi(\zeta), \varphi(a)) P_{a}(\zeta)|d \zeta| \rightarrow 0$ as $|a| \rightarrow 1$.

For the proof we assume that $a \in \mathbb{D}$ satisfies $2^{-N} \leq 1-|a|<2^{1-N}$ for some $N \geq 1$, and then let $N \rightarrow \infty$ in our estimates. Define for $k=1, \ldots, N$ the radii $r_{k}$, points $a_{k} \in \mathbb{D}$ and $\operatorname{arcs} I_{k}$ through $1-r_{k}=2^{N-k}(1-|a|), a_{k}=r_{k} a /|a|$ and $I_{k}=I\left(a_{k}\right)$. Set also $a_{0}=0$ and $I_{0}=\mathbb{T}$. Then $a=a_{N}$ and $I(a)=I_{N} \subset I_{N-1} \subset \ldots \subset I_{0}=\mathbb{T}$. Moreover, $2^{-k} \leq\left|I_{k}\right|<2^{1-k}$. Observe that if $1 \leq k<N$ and $\zeta \in I_{k} \backslash I_{k+1}$, then elementary trigonometry yields $|\zeta-a| \geq \frac{1}{2}\left|I_{k+1}\right| \geq 2^{-k-2}$. Hence the Poisson kernel satisfies $P_{a}(\zeta) \lesssim 2^{2 k-N}$ for all $\zeta \in I_{k} \backslash I_{k+1}$, where $\lesssim$ indicates that the left-hand side is bounded above by a constant multiple of the right-hand side, the constant being independent of $N$ and $k$. Consequently we may estimate the second integral appearing in Claim 2 as follows:

$$
\begin{aligned}
\int_{\mathbb{T}} \tau & (\varphi(\zeta), \varphi(a)) P_{a}(\zeta)|d \zeta| \\
& \lesssim \sum_{k=0}^{N-1} 2^{2 k-N} \int_{I_{k} \backslash I_{k+1}} \tau(\varphi(\zeta), \varphi(a))|d \zeta|+2^{N} \int_{I(a)} \tau(\varphi(\zeta), \varphi(a))|d \zeta| \\
& \lesssim \sum_{k=0}^{N} \frac{2^{k-N}}{\left|I_{k}\right|} \int_{I_{k}} \tau(\varphi(\zeta), \varphi(a))|d \zeta| \\
& \leq \sum_{k=0}^{N} \frac{2^{k-N}}{\left|I_{k}\right|} \int_{I_{k}} \tau\left(\varphi(\zeta), \varphi\left(a_{k}\right)\right)|d \zeta|+\sum_{k=0}^{N-1} 2^{k-N} \tau\left(\varphi\left(a_{k}\right), \varphi(a)\right) \\
& \equiv A_{N}+B_{N}
\end{aligned}
$$

It will suffice to verify that the condition of Claim 1 implies that the terms $A_{N}$ and $B_{N}$ both tend to zero as $N \rightarrow \infty$. First of all (observe that now (5.1) holds),

$$
\begin{aligned}
A_{N} & \lesssim\left(\sum_{k=0}^{[N / 2]} 2^{k-N}+\sum_{k=[N / 2]+1}^{N} 2^{k-N}\right) \frac{1}{\left|I_{k}\right|} \int_{I_{k}} \tau\left(\varphi(\zeta), \varphi\left(a_{k}\right)\right)|d \zeta| \\
& \lesssim N \cdot 2^{-N / 2}+\sup _{k>[N / 2]} \frac{1}{\left|I_{k}\right|} \int_{I_{k}} \tau\left(\varphi(\zeta), \varphi\left(a_{k}\right)\right)|d \zeta|
\end{aligned}
$$

Above the first term tends to zero trivially, and the second term by Claim 1, as $N \rightarrow \infty$. 
In order to relate the term $B_{N}$ to the averages in Claim 1 we introduce the shorthand $b_{k}=\left|I_{k}\right|^{-1} \int_{I_{k}} \tau\left(\varphi(\zeta), \varphi\left(a_{k}\right)\right)|d \zeta|$. Let $1 \leq k \leq N$. By averaging over the arc $I_{k}$ we get from the triangle inequality for $\tau$ that

$$
\begin{aligned}
\tau\left(\varphi\left(a_{k-1}\right), \varphi\left(a_{k}\right)\right) & \leq \frac{1}{\left|I_{k}\right|} \int_{I_{k}} \tau\left(\varphi(\zeta), \varphi\left(a_{k-1}\right)\right)|d \zeta|+\frac{1}{\left|I_{k}\right|} \int_{I_{k}} \tau\left(\varphi(\zeta), \varphi\left(a_{k}\right)\right)|d \zeta| \\
& \leq 2 b_{k-1}+b_{k},
\end{aligned}
$$

since $\left|I_{k-1}\right| \leq 2\left|I_{k}\right|$. Because $a=a_{N}$, we deduce that

$$
\tau\left(\varphi\left(a_{k}\right), \varphi(a)\right) \lesssim \sum_{j=k}^{N} b_{j} \leq(N-k+1) \max _{k \leq j \leq N} b_{j} .
$$

Put $E_{k}=\max _{k \leq j \leq N} b_{j}$, so that by combining the above estimates one has

$$
B_{N} \lesssim \sum_{k=0}^{N-1}(N-k+1) 2^{k-N} E_{k}
$$

where the $E_{k}$ 's have a uniform upper bound (independent of $a$ ) and $E_{[N / 2]} \rightarrow 0$ as $N \rightarrow \infty$. By splitting the preceding sum as before at the level $[N / 2]$ we deduce that $B_{N} \rightarrow 0$ as $N \rightarrow \infty$. This completes the proof of Claim 2 and hence of Proposition 16.

Remark 18. (1) The equivalence of conditions (ii)-(iii) in Theorem 13 can be proved without relying on the work of Section 2. One essentially argues as in the proof of Proposition 8 and invokes Lemma 15 together with the comments preceding it. Instead of using the Bessaga-Pełczyński selection principle one may just apply Proposition 6 twice, the second time to the image sequence. We leave the details to the interested reader.

(2) In $[\mathrm{MT}$ an analytic map $\varphi: \mathbb{D} \rightarrow \mathbb{D}$ is said to belong to the hyperbolic class $V M O A^{h}$ if $\varphi$ satisfies (iv'). Similarly, we may say that $\varphi$ belongs to the pseudohyperbolic class $V M O A^{p h}$ if (iv) holds. Thus Proposition 16 (and its converse) states that $\varphi \in V M O A^{h}$ if and only if $\varphi \in V M O A^{p h}$, which is an interpretation independent of composition operators.

(3) In the formulation of conditions (iv') and (iv) the metrics $\rho$ and $\tau$ are raised to different powers. However, in each condition the power is irrelevant. Namely, an inspection of the proof of Proposition 16 shows that one may replace $\tau$ by any power $\tau^{p}$ with $p>0$ in $(\overrightarrow{i v})$. This yields the same conclusion for condition $\left(\mathrm{v}^{2}\right)$, and the analogous fact for (iv) and ( (v) is obvious.

(4) Proposition 16 suggests the following problem, which we did not pursue any further: is there a version of the proposition for composition operators $\mathcal{B} \rightarrow B M O A$ ? We recall here that Xiao $[\mathrm{X}]$ (cf. also [LMT]) showed that $C_{\varphi}$ is compact $\mathcal{B} \rightarrow B M O A$ if and only if

$$
\lim _{r \rightarrow 1} \sup _{a \in \mathbb{D}} \int_{\{z:|\varphi(z)|>r\}} \frac{\left|\varphi^{\prime}(z)\right|^{2}}{\left(1-|\varphi(z)|^{2}\right)^{2}}\left(1-\left|\sigma_{a}(z)\right|^{2}\right) d A(z)=0,
$$

where $A$ is the planar Lebesgue measure. 


\section{REFERENCES}

[AK] F. Albiac and N.J. Kalton, Topics in Banach Space Theory, Springer, 2006.

[AFP] J. Arazy, S.D. Fisher and J. Peetre, Möbius invariant function spaces, J. Reine Angew. Math. 363 (1985), 110-145.

[AGL] R. Aron, P. Galindo and M. Lindström, Compact homomorphisms between algebras of analytic functions, Studia Math. 123 (1997), 235-247.

[BCM] P.S. Bourdon, J.A. Cima and A.L. Matheson, Compact composition operators on BMOA, Trans. Amer. Math. Soc. 351 (1999), 2183-2196.

[CRU] B.R. Choe, W Ramey and D. Ullrich, Bloch-to-BMOA pullbacks on the disk, Proc. Amer. Math. Soc. 125 (1997), 2987-2996.

[CM] J.A. Cima and A.L. Matheson, Weakly compact composition operators on VMO, Rocky Mountain J. Math. 32 (2002), 937-951.

[CMc] C.C. Cowen and B.D. MacCluer, Composition Operators on Spaces of Analytic Functions, CRC Press, 1995.

[G] D. Girela, Analytic functions of bounded mean oscillation, Complex Function Spaces (Mekrijärvi, 1999), Univ. Joensuu Dept. Math. Rep. Ser., 4, Univ. Joensuu, 2001, pp. 61-170.

[L1] J. Laitila, Weakly compact composition operators on vector-valued BMOA, J. Math. Anal. Appl., 308 (2005), 730-745.

[L2] J. Laitila, Composition operators and vector-valued BMOA, Integral Equations Operator Theory, 58 (2007), 487-502.

[L3] J. Laitila, Weighted composition operators on BMOA, Comput. Methods Funct. Theory 9 (2009), 27-46.

[Le] M.V. Lerbov, Subspaces of the VMO space (Russian), Teor. Funktsiı Funktsional. Anal. i Prilozhen. 46 (1986), 51-54; English transl. in J. Soviet Math. 48 (1990), 536-538.

[LMT] M. Lindström, S. Makhmutov and J. Taskinen, The essential norm of a Bloch-to- $Q_{p}$ composition operator, Canad. Math. Bull. 47 (2004), 49-59.

[LST] P. Liu, E. Saksman and H.-O. Tylli, Small composition operators on analytic vector-valued function spaces, Pacific J. Math. 184 (1998), 295-309.

[MM] K. Madigan and A. Matheson, Compact composition operators on the Bloch space, Trans. Amer. Math. Soc. 347 (1995), 2679-2687.

[MT] S. Makhmutov and M. Tjani, Composition operators on some Möbius invariant Banach spaces, Bull. Austral. Math. Soc. 62 (2000), 1-19.

[M] P.F.X. Müller, Isomorphisms between $H^{1}$ Spaces, Monografie Matematyczne, vol. 66, Birkhäuser, 2005.

[MS] P.F.X. Müller and G. Schechtman, On complemented subspaces of $H^{1}$ and VMO, Geometric Aspects of Functional Analysis (1987-88), Lecture Notes in Math., vol. 1376, Springer, 1989, pp. 113-125.

[S] D. Sarason, Weak compactness of holomorphic composition operators on $H^{1}$, Functional Analysis and Operator Theory (New Delhi, 1990), Lecture Notes in Math., vol. 1511, Springer, 1992, pp. 75-79.

[Sh] J.H. Shapiro, Composition Operators and Classical Function Theory, Springer, 1993.

[Sm] W. Smith, Compactness of composition operators on BMOA, Proc. Amer. Math. Soc. 127 (1999), 2715-2725.

[SZ] W. Smith and R. Zhao, Composition operators mapping into the $Q_{p}$ spaces, Analysis 17 (1997), 239-263.

[St] K. Stephenson, Weak subordination and stable classes of meromorphic functions, Trans. Amer. Math. Soc. 262 (1980), 565-577.

[T] M. Tjani, Compact composition operators on some Möbius invariant Banach spaces, Ph.D. Thesis, Michigan State University, 1996.

[Wi] I. Wik, On John and Nirenberg's theorem, Ark. Mat. 28 (1990), 193-200.

[WX] K.J. Wirths and J. Xiao, Global integral criteria for composition operators, J. Math. Anal. Appl. 269 (2002), 702-715. 
[W] H. Wulan, Compactness of composition operators on BMOA and VMOA, Sci. China Ser. A 50 (2007), 997-1004.

[WZZ] H. Wulan, D. Zheng and K. Zhu, Compact composition operators on BMOA and the Bloch space, Proc. Amer. Math. Soc. 137 (2009), 3861-3868.

[X] J. Xiao, Composition operators: $N_{\alpha}$ to the Bloch space to $Q_{p}$, Studia. Math. 139 (2000), 245-260.

[Y] S. Yamashita, Holomorphic functions of hyperbolically bounded mean oscillation, Bol. U.M.I. 5-B (1986), 983-1000.

[Z] K. Zhu, Operator Theory in Function Spaces, Dekker, 1990; 2nd ed. by Amer. Math. Soc., 2007.

Institute for Social and Economic Research, University of Essex, Colchester CO4 3SQ, United Kingdom

E-mail address: jlaitila@essex.ac.uk

Department of Mathematics and Statistics, University of Helsinki, PO Box 68, Fi00014 Helsinki, Finland

E-mail address: pjniemin@cc.helsinki.fi

Department of Mathematics and Statistics, University of Helsinki, PO Box 68, Fi00014 Helsinki, Finland

E-mail address: eero.saksman@helsinki.fi

Department of Mathematics and Statistics, University of Helsinki, PO Box 68, Fi00014 Helsinki, Finland

E-mail address: hojtylli@cc.helsinki.fi 